\title{
O Método do Elemento Finito Aplicado ao Estudo de Colisões Atômicas e Moleculares
}

\author{
Marcilio N. Guimarães, \\ Centro de Formação de Professores - UFRB, \\ 45300-000, Amargosa, BA \\ E-mail: marcilio@ufrb.edu.br \\ Frederico V. Prudente \\ Instituto de Física - UFBA \\ 40210-340, Salvador, BA \\ E-mail: prudente@ufba.br
}

Resumo: Este trabalho tem como objetivo desenvolver e aplicar um método teórico e computacional eficiente para o estudo quântico não-relativístico de colisões atômicas e moleculares. $O$ método é baseado no formalismo variacional da matriz $R$ e no método do elemento finito para expansão da função de onda em termos de um conjunto finito de funções de base locais, que apresenta como vantagem a possibilidade do desenvolvimento de um algoritmo de inversão de matrizes que reduz significativamente o tempo computacional no cálculo da matriz $R$. Calculamos o comprimento de espalhamento para a colisão elástica entre os átomos de rubídio e césio, e as probabilidades de transição no processo colinear $\mathrm{H}+\mathrm{H}_{2} \longrightarrow \mathrm{H}_{2}+\mathrm{H}$. A eficiência da metodologia utilizada foi avaliada comparando nossos resultados com diversos outros previamente publicados na literatura.

Palavras-chave: Elemento Finito, Matriz R, Colisões Atômicas e Moleculares

\section{Introdução}

O estudo do processo de colisão entre átomos e moléculas é fundamental para o entendimento de um enorme conjunto de fenômenos que englobam desde a química atmosférica e o processo de combustão química até fenômenos que ocorrem em meio estelar ou em gases ultrafrios. Ao contrário de sistemas ligados onde as partículas do sistema estão restritas em uma região do espaço, em processos de colisão as partículas do sistema estão livres para se propagar em toda a região do espaço de configurações e não busca-se determinar as energias, que assume valores contínuos ao invés de discretos, sendo agora uma condição inicial do problema. Neste caso deseja-se obter as quantidades físicas assintóticas como seção de choque e probabilidades de transição.

Nosso objetivo nesse trabalho é desenvolver e aplicar um método teórico e computacional eficiente para o estudo quântico não-relativístico de colisões atômicas e moleculares. O método é baseado no formalismo variacional da matriz $R$ para estados não ligados, que será apresentado na seção 2. Também empregamos o método do elemento finito (MEF), a ser apresentado na seção 3, para expansão da função de onda em termos de um conjunto finito de funções de base locais, que possibilita o desenvolvimento de um algoritmo de inversão de matrizes reduzindo significativamente o tempo computacional no cálculo da matriz $R$. As aplicações são apresentadas na seção 4 onde calculamos o comprimento de espalhamento da colisão fria entre o Rubídio e o Césio. Adicionalmente, calculamos as probabilidades de transição, para a reação colinear $\mathrm{H}+\mathrm{H}_{2} \longrightarrow \mathrm{H}_{2}+\mathrm{H}$. 


\section{Problema Molecular}

Operador hamiltoniano para um sistema com $N$ partículas de massa $M_{j}$ é dado por

$$
\widehat{H}=-\frac{\hbar^{2}}{2} \sum_{j=1}^{N} \frac{1}{M_{j}} \nabla_{j}^{2}+V(\mathbf{x}),
$$

onde $V(\mathbf{x})$ é a superfície de energia potencial. Resolver a equação de Schrödinger é equivalente a encontrar o extremo do seguinte funcional

$$
J[\Psi]=\int \Psi^{*}(\mathbf{x})\{\widehat{H}-E\} \Psi(\mathbf{x}) d \nu,
$$

onde devemos expandir a função de onda, $\Psi(\mathbf{x})$, em um conjunto de funções de base $\left\{f_{i}\right\}$.

No caso de sistemas ligados a condição de contorno implica que a função de onda deve se anular numa superfície $\mathcal{A}$. Por outro lado, as propriedades físicas que caracterizam os estados de espalhamento conduzem a uma condição de contorno diferente. Formalmente, esta diferença na teoria de espalhamento é evidenciada dividindo o espaço das coordenadas em duas regiões: a região exterior, ou assintótica, em que a solução analítica da equação de Schrödinger é conhecida, e a região interior, ou de interação, onde o processo de colisão é essencialmente governado pelas forças de curto alcance entre as partículas. O formalismo variacional da matriz $R$ especifica que

$$
\Psi(\mathbf{x})=\int_{\mathcal{A}} d \mathbf{x}^{\prime} R\left(\mathbf{x} ; \mathbf{x}^{\prime}\right) \Psi_{S}^{\prime}\left(\mathbf{x}^{\prime}\right),
$$

onde $R\left(\mathbf{x} ; \mathbf{x}^{\prime}\right)$ relaciona os pontos $\mathbf{x}$ e $\mathbf{x}^{\prime}$ em $\mathcal{A}$ e é chamado de núcleo da matriz $R$ que está conectado à matriz $S$ que, por sua vez, está diretamente associada às quantidades física de interesse [10]. Assim, aplicando a condição acima na equação (2) obtemos a expressão para o núcleo da matriz $R$ dado por

$$
R\left(\mathbf{x} ; \mathbf{x}^{\prime}\right)=\frac{\hbar^{2}}{2} \mathbf{f}(\mathbf{x})[\mathbf{H}-E \mathbf{O}]^{-1} \mathbf{f}^{\dagger}\left(\mathbf{x}^{\prime}\right)
$$

onde $\mathbf{H}$ e $\mathbf{O}$ são as matrizes hamiltoniana e superposição, respectivamente, e $\mathbf{f}(\mathbf{x})$ é o vetor das funções de base $\left\{f_{i}\right\}$.

Notadamente, um grande esforço computacional é requerido, tanto no cálculo dos elementos da matriz $\mathbf{H}-E \mathbf{O}$ como na sua inversão para obtenção do núcleo da matriz $R$. Considerando que uma matriz tem dimensão $p \times p$, o esforço computacional na inversão cresce com $p^{3}$ [8]. Portanto, a eficiência do cálculo numérico dependerá de uma escolha correta das funções de base que serão utilizadas para expandir a função de onda do sistema. Como veremos na próxima seção, o método do elemento finito faz com que as matrizes $\mathbf{H}$ e $\mathbf{O}$ fiquem bastante esparsas e fornece um eficiente algoritmo de inversão de matrizes.

\section{Método do Elemento Finito}

O método do elemento finito (MEF) no caso unidimensional consiste em dividir o intervalo de integração $[a, b]$ em $N_{e}$ elementos, sendo o $i$-ésimo elemento definido no intervalo de $q_{i-1}$ até $q_{i}$ com $q_{0}=a$ e $q_{N_{e}}=b$, e a função de onda é expandida da seguinte forma:

$$
\Psi(q)=\sum_{i=1}^{N_{e}} \sum_{j=0}^{k_{i}} c_{j}^{i} f_{j}^{i}(q),
$$

onde as funções de base $\left\{f_{j}^{i}(q)\right\}$ satisfazem a propriedade $f_{j}^{i}(q)=0$ se $q \notin\left[q_{i-1}, q_{i}\right]$. O parâmetro $k_{i}$ é a mais alta ordem dos polinômios associados com $i$-ésimo elemento, $f_{j}^{i}(q)$ é a $j$-ésima função de base do mesmo elemento e $\left\{c_{j}^{i}\right\}$ são os coeficientes da expansão. 
A representação matricial $\mathbf{B}$ de qualquer operador local $\widehat{B}$ é obtida empregando-se o formalismo variacional. Devido ao fato das funções de base do MEF serem locais, tal representação matricial assume uma forma tridiagonal em blocos:

$$
\mathbf{B}=\left[\begin{array}{cccccc}
\mathbf{B}^{1} & \mathbf{b}^{1} & \mathbf{0} & \mathbf{0} & \cdots & \mathbf{0} \\
\left(\mathbf{b}^{1}\right)^{\dagger} & \mathbf{B}^{2} & \mathbf{b}^{2} & \mathbf{0} & \cdots & \mathbf{0} \\
\mathbf{0} & \left(\mathbf{b}^{2}\right)^{\dagger} & \mathbf{B}^{3} & \mathbf{b}^{3} & \ddots & \vdots \\
\mathbf{0} & \mathbf{0} & \left(\mathbf{b}^{3}\right)^{\dagger} & \ddots & \ddots & \mathbf{0} \\
\vdots & \vdots & \ddots & \ddots & \mathbf{B}^{N_{e}} & \mathbf{b}^{N_{e}} \\
\mathbf{0} & \mathbf{0} & \cdots & \mathbf{0} & \left(\mathbf{b}^{N_{e}}\right)^{\dagger} & \mathbf{B}^{N_{e}+1}
\end{array}\right]
$$

onde $\mathbf{B}^{i}$ e $\mathbf{b}^{i}$, com $i=1, \ldots N_{e}$, são submatrizes quadradas de dimensão $k_{i} \times k_{i}$ e

$$
\mathbf{B}^{N_{e}+1}=B_{k_{N_{e}} k_{N_{e}}}^{N_{e}} .
$$

Agora estamos interessados em desenvolver uma técnica para inverter a matriz $\mathbf{B} \equiv \mathbf{H}-E \mathbf{O}$ necessária para obtenção do núcleo da matriz $R$ conforme o formalismo variacional desenvolvido na seção 2. Conforme vimos, para o caso unidimensional, utilizando o MEF a matriz $\mathbf{B}$ assume uma estrutura em blocos dada pela expressão (6). Usando a notação do método do elemento finito, a inversa de $\mathbf{B}$ pode ser escrita como

$$
\mathbf{C}=\mathbf{B}^{-1}=\left[\begin{array}{cccc}
\mathbf{C}^{11} & \mathbf{C}^{12} & \cdots & \mathbf{C}^{1 N_{e}+1} \\
\mathbf{C}^{21} & \mathbf{C}^{22} & \cdots & \mathbf{C}^{2 N_{e}+1} \\
\vdots & \vdots & \ddots & \vdots \\
\mathbf{C}^{N_{e}+11} & \mathbf{C}^{N_{e}+12} & \cdots & \mathbf{C}^{N_{e}+1 N_{e}+1}
\end{array}\right]
$$

Como na $p$-MEF somente uma função de base que expande a função de onda será diferente de zero no último nó da malha o vetor das funções de base assumirá a forma

$$
\mathbf{f}\left(q_{N_{e}}\right)=\left[\begin{array}{llll}
0 & 0 & \cdots & 1
\end{array}\right]
$$

Devido a essas propriedades, utilizando a $p$-MEF para expandir a função de onda, obteremos o núcleo da matriz $R$ do sistema em questão, dada pela equação (4), como

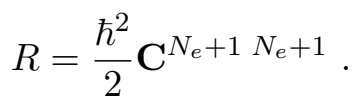

Portanto, precisamos apenas do último bloco da inversa da matriz $\mathbf{B}$ para obter o núcleo da matriz $R$. O processo de inversão que desenvolveremos a seguir visa calcular somente este último bloco da matriz inversa.

O método de partição de matrizes visa inicialmente inverter matrizes de ordem tão grande que todos os seus elementos não podem ser armazenados na memória do computador. Consideremos o problema de obter a inversa, $\mathbf{Y}$, de uma matriz quadrada $\mathbf{X}$ que é particionada como

$$
\mathbf{X}=\left[\begin{array}{ll}
\mathbf{X}_{11} & \mathbf{X}_{12} \\
\mathbf{X}_{21} & \mathbf{X}_{22}
\end{array}\right] \Rightarrow \mathbf{Y}=\mathbf{X}^{-1}=\left[\begin{array}{ll}
\mathbf{Y}_{11} & \mathbf{Y}_{12} \\
\mathbf{Y}_{21} & \mathbf{Y}_{22}
\end{array}\right]
$$

sendo $\mathbf{X}_{11}$ e $\mathbf{X}_{22}$ submatrizes quadradas e não-singulares, e as submatrizes de $\mathbf{Y}$ têm a mesma dimensão que as submatrizes de $\mathbf{X}$ com índice correspondente. Em particular o último bloco da matriz inversa é dado por

$$
\mathbf{Y}_{22}=\left(\mathbf{X}_{22}-\mathbf{X}_{21} \mathbf{X}_{11}^{-1} \mathbf{X}_{12}\right)^{-1}
$$


Aplicamos agora o procedimento de inversão de matriz por blocos para calcular o último bloco da inversa da matriz B. Sendo assim definimos:

$$
\begin{gathered}
\mathbf{X}_{11}=\left[\begin{array}{ccccc}
\mathbf{B}^{1} & \mathbf{b}^{1} & \mathbf{0} & \cdots & \mathbf{0} \\
\left(\mathbf{b}^{1}\right)^{\dagger} & \mathbf{B}^{2} & \mathbf{b}^{2} & \ddots & \vdots \\
\mathbf{0} & \left(\mathbf{b}^{2}\right)^{\dagger} & \ddots & \ddots & \mathbf{0} \\
\vdots & \ddots & \ddots & \mathbf{B}^{N_{e}-1} & \mathbf{b}^{N_{e}-1} \\
\mathbf{0} & \cdots & \mathbf{0} & \left(\mathbf{b}^{N_{e}-1}\right)^{\dagger} & \mathbf{B}^{N_{e}}
\end{array}\right] \quad, \quad \mathbf{X}_{22}=\mathbf{B}^{N_{e}+1} \\
\mathbf{X}_{12}=\left[\begin{array}{c}
\mathbf{0} \\
\mathbf{0} \\
\vdots \\
\mathbf{0} \\
\mathbf{b}^{N_{e}}
\end{array}\right] \quad \text { e } \mathbf{X}_{21}=\left[\begin{array}{lllll}
\mathbf{0} & \mathbf{0} & \cdots & \mathbf{0} & \left(\mathbf{b}^{N_{e}}\right)^{\dagger}
\end{array}\right] .
\end{gathered}
$$

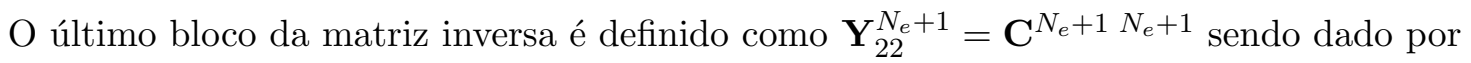

$$
\begin{aligned}
\mathbf{Y}_{22}^{N_{e}+1} & =\left(\mathbf{X}_{22}-\mathbf{X}_{21} \mathbf{X}_{11}^{-1} \mathbf{X}_{12}\right)^{-1} \\
& =\left(\mathbf{B}^{N_{e}+1}-\left(\mathbf{b}^{N_{e}}\right)^{\dagger} \mathbf{Y}_{22}^{N_{e}} \mathbf{b}^{N_{e}}\right)^{-1}
\end{aligned}
$$

onde $\mathbf{Y}_{22}^{N_{e}}$ é o último bloco da matriz inversa de $\mathbf{X}_{11}$. Portanto, podemos utilizar novamente o método de partição para obtermos este bloco. Para tanto redefinimos as matrizes $\mathbf{X}_{11}, \mathbf{X}_{22}$, $\mathbf{X}_{12}$ e $\mathbf{X}_{21}$ de modo que o último bloco da matriz inversa fica então dado por

$$
\begin{aligned}
\mathbf{Y}_{22}^{N_{e}} & =\left(\mathbf{X}_{22}-\mathbf{X}_{21} \mathbf{X}_{11}^{-1} \mathbf{X}_{12}\right)^{-1} \\
& =\left(\mathbf{B}^{N_{e}}-\left(\mathbf{b}^{N_{e}-1}\right)^{\dagger} \mathbf{Y}_{22}^{N_{e}-1} \mathbf{b}^{N_{e}-1}\right)^{-1}
\end{aligned}
$$

onde $\mathbf{Y}_{22}^{N_{e}-1}$ é o último bloco da inversa da matriz $\mathbf{X}_{11}$ redefinida. Assim, percebemos que, de forma geral, para se obter um bloco $\mathbf{Y}_{22}^{i}$ devemos calcular o bloco $\mathbf{Y}_{22}^{i-1}$ através da expressão geral:

$$
\mathbf{Y}_{22}^{i}=\left(\mathbf{B}^{i}-\left(\mathbf{b}^{i-1}\right)^{\dagger} \mathbf{Y}_{22}^{i-1} \mathbf{b}^{i-1}\right)^{-1}
$$

começando com $\mathbf{Y}_{22}^{1}=\left(\mathbf{B}^{1}\right)^{-1}$.

\section{Aplicações}

Todos os cálculos dessa seção foram feitos usando uma implementação computacional em Fortran baseada no formalismo variacional da matriz $R$ e na versão- $p$ do método do elemento finito ( $p$ MEF) apresentados nas seções 2 e 3, respectivamente. Também em todos os cálculos com a $p$-MEF a ordem dos polinômios empregada foi a mesma para todos os elementos da malha (i.e., $k_{i}=$ constante, $\left.\forall i\right)$.

Começamos a seção apresentando dados sobre o comprimento de espalhamento, $a$, para a colisão elástica entre o átomo de césio $\left({ }^{133} \mathrm{Cs}\right)$ e o átomo de rubídio $\left({ }^{85} \mathrm{Rb}\right.$ e $\left.{ }^{87} \mathrm{Rb}\right)$ frios com temperaturas próximas ao zero absoluto - interagindo via o estado fundamental $\left(X^{1} \Sigma^{+}\right)$ da molécula RbCs. Para uma onda parcial com $l=0$, resolvemos o problema de inversão de matriz dado pela equação (4) encontrando a matriz $R$ para essa onda parcial, e calculamos o deslocamento de fase $\delta_{0}$ e sua tangente (veja detalhes em [10]). Consideramos a energia, $E$, da ordem de $10^{-30}$ hartree garantindo um $k=\sqrt{2 \mu E / \hbar^{2}}$ (onde $\mu$ é a massa reduzida dos núcleos) bem pequeno de modo que $a$ é calculado pela sua definição dada pela equação

$$
-\frac{1}{a}=\lim _{k \rightarrow 0}\left[k \cot \delta_{0}\right]
$$


Tabela 1: Influência do valor da separação máxima, $r_{\max }$, na convergência do comprimento de espalhamento, $a$, ambos em bohr, para a colisão $\mathrm{Rb}-\mathrm{Cs}$ no estado $X^{1} \Sigma^{+}$, calculado com potencial de Jamieson et al [5]. Massa, em unidades de massa atômica: $m\left({ }^{133} \mathrm{Cs}\right)=132,905447$ u.a.; $m\left({ }^{85} \mathrm{Rb}\right)=84,9117893$ u.a.; $m\left({ }^{87} \mathrm{Rb}\right)=86,9091835$ u.a..

\begin{tabular}{lcc}
\hline \hline & ${ }^{85} \mathrm{Rb}-{ }^{133} \mathrm{Cs}$ & ${ }^{87} \mathrm{Rb}-{ }^{133} \mathrm{Cs}$ \\
$r_{\max }$ & $a$ & $a$ \\
\hline 200 & 67,6489 & 83,8008 \\
300 & 50,2289 & 69,5049 \\
400 & 44,5831 & 64,5238 \\
500 & 42,3731 & 62,5026 \\
600 & 41,3493 & 61,5461 \\
700 & 40,8135 & 61,0385 \\
800 & 40,5067 & 60,7450 \\
900 & 40,3188 & 60,5639 \\
1000 & 40,1972 & 60,4461 \\
1200 & 40,0579 & 60,3102 \\
1400 & 39,9862 & 60,2398 \\
1600 & 39,9456 & 60,1997 \\
1800 & 39,9208 & 60,1753 \\
2000 & 39,9050 & 60,1595 \\
6000 & 39,8634 & 60,1180 \\
\hline \hline
\end{tabular}

esse valor é, por exemplo, bem menor do que o valor considerado por Zanelatto et al [11] que considerou a energia da ordem de $10^{-13}$ hartree. Para alcançar uma boa acurácia nos resultados com o $p$-MEF foi usada uma malha equidistante com $N_{e}=60$ no intervalo $[0,40]$ bohr, $N_{e}=50$ no intervalo [40, $\left.r_{\max }\right]$ bohr e $k_{i}=30$, garantindo um fator de convergência, $\Delta a=$ $\left|a\left(N_{e}, k_{i}\right)-a\left(N_{e}, k_{i-1}\right)\right|$, de pelos menos cinco casas decimais, onde $a\left(N_{e}, k_{i}\right)$ é o comprimento de espalhamento como função dos parâmetros da base $\left(N_{e}\right.$ e $\left.k_{i}\right)$. A dimensão da matriz $\mathbf{B}=\mathbf{H}-E \mathbf{O}$ é $\left(N_{e} \cdot k_{i}+1\right) \times\left(N_{e} \cdot k_{i}+1\right)$ e seu último bloco tem dimensão 1 .

Utilizamos a curva de energia potencial proposto por Jamieson et al [5] que usaram seus dados de curto alcance calculados ab initio conectando-o, em 17,9524 bohr, à expressão analítica para longo alcance. A fim de fazer uma conexão suave entre as duas partes do potencial juntamos o valor do potencial de longo alcance em 17,9524 bohr aos pontos calculados ab initio utilizando um esquema de interpolação do potencial de curto alcance por spline cúbica. Jamieson et al [5] usaram o método de Numerov para resolver a equação de Schrödinger radial para pequenos valores assintóticos do número de onda, $k$, determinando o comprimento de espalhamento a partir de uma expansão. Empregando seu potencial proposto, eles obtiveram o valor de $a=$ 40, 24 bohr, para a colisão ${ }^{85} \mathrm{Rb}-{ }^{133} \mathrm{Cs}$, e, $a=60,18 \mathrm{bohr}$, para a colisão ${ }^{87} \mathrm{Rb}-{ }^{133} \mathrm{Cs}$. Por sua vez, Zanelatto et al [11] também usaram o método de Numerov para determinar o comprimento de espalhamento. Empregando o mesmo potencial, eles obtiveram o valor de $a=40,357 \mathrm{bohr}$, para a colisão ${ }^{85} \mathrm{Rb}-{ }^{133} \mathrm{Cs}$, e, $a=60,610 \mathrm{bohr}$, para a colisão ${ }^{87} \mathrm{Rb}-{ }^{133} \mathrm{Cs}$. Também empregando o mesmo potencial, mostramos na Tabela 1 a influência do valor da separação máxima, $r_{\max }$, na convergência do comprimento de espalhamento para as colisões ${ }^{85} \mathrm{Rb}-{ }^{133} \mathrm{Cs}$ e ${ }^{87} \mathrm{Rb}-{ }^{133} \mathrm{Cs}$. Podemos notar na tabela que os presentes resultados convergem para um valor bastante próximo dos obtidos por Jamieson et al e Zanelatto et al para uma larga separação máxima; a melhor concordância é alcançada em torno de $r_{\max }=1000$ bohr.

Agora testamos a metodologia computando as probabilidades de transição para uma reação 
Tabela 2: Probabilidades de transição inelástica e reativa selecionadas $\left(\nu_{I} \rightarrow \nu_{F}\right)$ em algumas energias, $E$, para o sistema $\mathrm{H}+\mathrm{H}_{2}$ ena superfície de energia potencial LSTH. Nossos presentes resultados com a $p$-MEF usam $\rho_{\max }=20$ bohr, $N_{e}=200$ e $k=6$.

\begin{tabular}{ccccc}
\hline \hline \multicolumn{2}{c}{ Transição } & $E[\mathrm{eV}]$ & Presente & SKV $^{\dagger}$ \\
\hline Inelástica & $0 \rightarrow 0$ & 0,500 & 0,917 & 0,917 \\
& & 1,100 & 0,171 & 0,172 \\
& & 1,400 & 0,299 & 0,300 \\
& \multirow{4}{*}{$1 \rightarrow 1$} & 0,800 & $6,17 \times 10^{-2}$ & $6,22 \times 10^{-2}$ \\
& & 1,100 & 0,123 & 0,123 \\
Reativa & $2 \rightarrow 2$ & 1,400 & $8,70 \times 10^{-2}$ & $8,74 \times 10^{-2}$ \\
& $0 \rightarrow 1$ & 1,100 & 0,158 & 0,157 \\
& & 1,400 & 0,381 & 0,380 \\
& & 0,800 & $4,22 \times 10^{-5}$ & 0,224 \\
& & 1,100 & 0,344 & $0,74 \times 10^{-5}$ \\
& $0 \rightarrow 2$ & 1,400 & 0,304 & 0,305 \\
& $1 \rightarrow 2$ & 1,400 & 0,107 & 0,107 \\
$2 \rightarrow 2$ & 1,400 & 0,125 & 0,126 \\
& $2 \rightarrow 1$ & 0,482 & 0,482 \\
\hline \hline
\end{tabular}

${ }^{\dagger}$ Resultados do princípio variacional de Kohn da matriz $S$ [6]

colinear. Restringimos nossa atenção em processos químicos do tipo

$$
H+H_{2}\left(\nu_{I}\right) \longrightarrow\left\{\begin{array}{cc}
H+H_{2}\left(\nu_{F}\right) & (\text { elástico/inelástico) } \\
H_{2}\left(\nu_{F}\right)+H & \text { (reativo) }
\end{array} .\right.
$$

usando a superfície de energia potencial LSTH [9]. A formulação variacional adiabática do problema empregando coordenadas hiperesféricas permite efetuar os cálculos em duas etapas (veja detalhes em [2]). Primeiro diagonalizamos uma matriz a fim de encontrarmos os autovalores e autofunções do problema hiperangular para cada valor do hiperraio. Para isso usamos a $p$ MEF unidimensional com a malha otimizada [7]. A segunda etapa é a obtenção da matriz $R$ hiperesférica usando os valores obtidos no problema hiperangular. Para fazer isso, nós usamos a $p$-MEF com malha uniforme e a técnica de inversão de matrizes vista na seção 3. Neste caso, a matriz $\mathbf{B}=\mathbf{H}-E \mathbf{O}$ tem dimensão $\left(N_{e} \cdot k_{i}+1\right) \cdot n \times\left(N_{e} \cdot k_{i}+1\right) \cdot n$ e seu último bloco tem dimensão $n \times n$, onde $n$ é o número de funções hiperangulares [2]. Por fim, as probabilidades de transição $P_{\nu_{I} \nu_{F}}(E)=\left|S_{\nu_{I} \nu_{F}}\right|^{2}$ são computadas a partir da matriz $S$ que está diretamente relacionada com com a matriz $R$.

Para checar a acurácia da metodologia, na Tabela 2 é mostrado algumas probabilidades de transição elástica e inelástica para diferentes valores de energia e comparadas com os resultados acurados obtidos usando um método independente do tempo baseado no princípio variacional de Kohn da matriz $S$ (SKV) [6]. Em nossos resultados apresentados foi escolhido um hiperraio máximo de 20 bohr com o parâmetro $N_{e}=200$ e $k_{i}=6$ para cálculos. Note que eles estão em excelente concordância com os resultados acurados usando a SKV. As diferenças entre nossos resultados são comparáveis com as diferenças entre os resultados SKV e os cálculos utilizando outros métodos tal como a aproximação de Hartree dependente do tempo de multi configuração [4], o formalismo do pacote de onda independente do tempo [3] e a formulação do pacote de onda real dependente do tempo [1].

\section{Conclusão}

Observamos, de modo geral, que nossos resultados concordaram com outros bastante precisos previamente publicados. Apontamos que o procedimento é suficientemente acurado mesmo em 
distâncias interatômicas muito grandes, tipicamente da ordem de milhares de bohr. Também apontamos que as matrizes hamiltoniana e superposição precisam ser construídas somente uma vez e então, armazenadas na memória do computador, podem ser utilizadas para calcular as informações de espalhamento em qualquer energia. Além disso, a presente metodologia permite determinar todas as probabilidades de transição reativa e inelástica em um único cálculo para cada energia, distinto dos métodos dependentes do tempo onde usualmente é necessário realizar diversas propagações do pacote de onda. Enfatizamos que, usando as propriedades da $p$-MEF, podemos implementar um eficiente algoritmo para obtenção da matriz $R$ baseado numa técnica de inversão de matriz que usa pequenos blocos da matriz, onde são necessários manter na memória do computador apenas os blocos não nulos da matriz. A vantagem é que isso reduz o esforço requerido para inversão da matriz, que geralmente é bastante grande em aproximações variacionais.

\section{Referências}

[1] S. K. Gray and G. G. Balint-Kurti, Quantum dynamics with real wave packets, including application to three-dimensional $(J=0) \mathrm{D}+\mathrm{H}_{2} \longrightarrow \mathrm{HD}+\mathrm{H}$ reactive scattering, $J$. Chem. Phys., 108 (1998) 950.

[2] M. N. Guimarães e F. V. Prudente, A variational adiabatic hyperspherical finite element $R$ matrix methodology: General formalism and application to $\mathrm{H}+\mathrm{H}_{2}$ reaction, Eur. Phys. J. D., 64 (2011) 287.

[3] Y. Huang, S. S. Iyengar, D. J. Kouri e D. K. Hoffman, Further analysis of solutions to the time-independent wave packet equations of quantum dynamics. II. Scattering as a continuous function of energy using finite, discrete approximate Hamiltonians, J. Chem. Phys., 105 (1996) 927.

[4] A. Jäckle e H.-D. Meyer, Reactive scattering using the multiconfiguration time-dependent Hartree approximation: General aspects and application to the collinear $\mathrm{H}+\mathrm{H}_{2} \longrightarrow \mathrm{H}_{2}+\mathrm{H}$ reaction, J. Chem. Phys., 102 (1995) 5605.

[5] M. J. Jamieson, H. Sarbazi-Azad, H. Ouerdane, G.-H. Jeung, Y. S. Lee e W. C. Lee, Elastic scattering of cold caesium and rubidium atoms, J. Phys. B: At. Mol. Opt. Phys., 36 (2003) 1085 .

[6] W. H. Miller, Recent advances in quantum mechanical reactive scattering theory, including comparison of recent experiments with rigorous calculations of state-to-state cross section for the $\mathrm{H} / \mathrm{D}+\mathrm{H}_{2} \longrightarrow \mathrm{H}_{2} / \mathrm{HD}+\mathrm{H}$ reactions, Annu. Rev. Phys. Chem, 41 (1990) 245.

[7] E. M. Nascimento, F. V. Prudente, M. N. Guimarães e Maniero A. M., A study of the electron structure of endohedrally confined atoms using a model potential, J. Phys. B: At. Mol. Opt. Phys, 44 (2011) 015003.

[8] W. H Press, S. A Teukolsky, W. T. Vetterling e B. P. Flannery, "Numerical Recipes in Fortran 77: The Art of Scientific Computing", Cambridge University Press, New York, 1992.

[9] D. G. Truhlar e C. J. Horowitz, Functional representation of Liu and Siegbahn's accurate ab initio potential energy calculations for $\mathrm{H}+\mathrm{H}_{2}$, J. Chem. Phys., 68 (1978) 2466.

[10] T. Y. Wu e T. Ohmura, "Quantum Theory of Scattering", Prentice-Hall, Englewwood Cliffs, 1962.

[11] A. L. M. Zanelatto, E. M. S. Ribeiro e R. d. J. Napolitano, Scattering lengths for Li-Cs, $\mathrm{Na}-\mathrm{Cs}, \mathrm{K}-\mathrm{Cs}$ and Rb-Cs ultracold collisions, J. Chem. Phys., 123 (2005) 014311. 